IOS Press

\title{
Expression of FOXM1 and Aurora-A predicts prognosis and sorafenib efficacy in patients with hepatocellular carcinoma
}

\author{
Wen-Lung Su ${ }^{\mathrm{a}}$, Shih-Chang Chuang ${ }^{\mathrm{b}}$, Yu-Chu Wang ${ }^{\mathrm{b}}$, Lin-An Chen ${ }^{\mathrm{c}}$, Jian-Wei Huang ${ }^{\mathrm{b}}$, \\ Wen-Tsan Chang ${ }^{\mathrm{b}}$, Shen-Nien Wang ${ }^{\mathrm{b}}$, King-Teh Lee ${ }^{\mathrm{b}}$, Chang-Shen Lin ${ }^{\mathrm{d}}$ and Kung-Kai Kuo ${ }^{\mathrm{b}, *}$ \\ ${ }^{a}$ Department of Surgery, Kaohsiung Municipal Ta-Tung Hospital, Kaohsiung, Taiwan \\ ${ }^{\mathrm{b}}$ Division of General and Digestive Surgery, Department of Surgery, Kaohsiung Medical University Hospital, \\ Kaohsiung Medical University, Kaohsiung, Taiwan \\ ${ }^{\mathrm{c}}$ Department of Surgery, Health and Welfare Ministry Pingtung Hospital, Pingtung, Taiwan \\ ${ }^{\mathrm{d}}$ Graduate Institute of Medicine, College of Medicine, Kaohsiung Medical University, Kaohsiung, Taiwan
}

\begin{abstract}
.
BACKGROUND: Effective prognostic biomarkers and powerful target-therapeutic drugs are needed for improving the treatment of Hepatocellular carcinoma (HCC).

OBJECTIVE: This study aimed to evaluate the expression of FOXM1 and Aurora-A and their prognostic value in HCC.

METHODS: We determined the differentially expressed genes signature in HCC using the Gene Set Enrichment Analysis (GSEA), and then evaluated the expression of FOXM1 and Aurora-A in TCGA and KMUH cohort. Associations between co-expression of FOXM1 and Aurora-A and clinical variables were calculated. Overall survival (OS) and recurrence-free survival (RFS) were estimated with different FOXM1 and Aurora-A expression status.

RESULTS: FOXM1-related gene sets were mostly associated with cell cycle regulation in HCC tissues. We found a positive correlation between the expression of FOXM1 and Aurora-A. Overexpression of FOXM1 and Aurora-A was associated with larger tumor size, advanced stage, higher grade, and double-positive for HBV and HCV. The coordinated overexpression of FOXM1 and Aurora-A was the most significant independent prognostic factor for OS and RFS. Furthermore, the concomitant high expression of FOXM1 and Aurora-A predicted the worst OS of sorafenib-treated patients with HCC.

CONCLUSIONS: The co-expression of FOXM1 and Aurora-A could be a reliable biomarker to predict the sorafenib response and prognosis of HCC patients.
\end{abstract}

Keywords: FOXM1, Aurora-A, hepatocellular carcinoma (HCC), sorafenib

\section{Introduction}

Hepatocellular carcinoma (HCC) usually results in a high mortality rate, especially in South-Eastern Asia [1]. Some therapeutic strategies such as curative resection, liver transplantation, and target therapy may

\footnotetext{
${ }^{*}$ Corresponding author: Kung-Kai Kuo, Division of General and Digestive Surgery, Department of Surgery, Kaohsiung Medical University Hospital, Kaohsiung Medical University, No. 100, Tz-You 1st Rd, San-Ming District, Kaohsiung, 807, Taiwan. Tel.: +88673121101 ext. 7651; Fax: +886 7 3216992; E-mail: kuoksfo168@gmail.com.
}

be promising for the treatment of $\mathrm{HCC}$, whereas its prognosis remains unsatisfactory due to frequent recurrence and metastasis rates. HCC is a highly heterogeneous malignancy with robust clinical and molecular diversities. Many HCC patients are diagnosed with advanced-stage due to the lack of precise early diagnosis and effective treatment [2]. HCC treatment failures are primarily caused by frequent recurrence and lead to poor prognosis. New reliable biomarkers for survival prognosis and prediction of drug response are needed because they are crucial for developing more effective diagnostic and therapeutic strategies. 
Many genome-wide expression profiling studies have revealed genetic alterations during tumorigenesis in the past decade. The combination of transcriptome sequencing and bioinformatics analysis has allowed the identification of differentially expressed genes involved in the development and progression of HCC. Although serum alfa-fetoprotein (AFP) can be a diagnostic marker to detect HCC, normal AFP levels are present in $30 \%$ of patients at the time of diagnosis, even with advanced HCC $[3,4]$. However, there is still no reliable biomarker to predict the survival prognosis of HCC by liver biopsy in clinical practice. Thus, it is essential to find new putative biomarkers that may predict the prognosis of HCC and guide personalized therapy for HCC patients.

Forkhead box protein M1 (FOXM1) plays an important role in regulating cell cycle progression, differentiation of cancer, and drug resistance [5]. Recent studies demonstrated that FOXM1 is a key transcription factor associated with HCC [6] and that elevated FOXM1 expression is associated with poor prognosis of HCC [7], colorectal cancer [8] and lung cancer [9]. FOXM1 also is related to drug resistance in breast and lung cancer $[10,11]$. These results clearly show the critical role of FOXM1 in tumorigenesis and therapeutic response to chemotherapy. Although FOXM1 is upregulated in numerous cancers, it is unclear whether the differentially expressed genes signature, including FOXM1, can be prognostic biomarkers and whether they contribute to alternative signaling pathways for the tumor progression of HCC.

Here, we identify specific genes that are significantly co-deregulated with FOXM1 in HCC. We analyzed the expression data of the HCC cohort from The Cancer Genome Atlas (TCGA) and a validation (KMUH) cohort and evaluated an association between the gene expression signature and clinicopathological characteristics, including patient's survival. FOXM1 can predict the worst overall survival (OS) of sorafenib-treated patients with HCC. This work will provide further insight into developing prognostic biomarkers and potential molecular targets for new therapeutic direction.

\section{Materials and methods}

\subsection{The Cancer Genome Atlas (TCGA) dataset}

We downloaded TCGA Liver Cancer (LIHC) level 3 processed mRNA expression data and clinicopathological data from the UCSC Xena (http://xena.ucsc.edu).
Table 1

Clinical variables of the HCC patients

\begin{tabular}{cc}
\hline Variables & No. $(\%)(n=341)$ \\
\hline Age & $151(44.3 \%)$ \\
$<60$ & $190(55.7 \%)$ \\
$\geqslant 60$ & \\
Gender & $231(67.7 \%)$ \\
Male & $110(32.3 \%)$ \\
Female & \\
TNM stage & $253(74.6 \%)$ \\
T1-2 & $86(25.4 \%)$ \\
T3-4 & $231(98.3 \%)$ \\
N0 & $4(1.7 \%)$ \\
N1 & $245(98.8 \%)$ \\
M0 & $3(1.2 \%)$ \\
M1 & \\
Stage & $237(74.3 \%)$ \\
$1-2$ & $82(25.7 \%)$ \\
$3-4$ & \\
Grade & $213(63.4 \%)$ \\
$1-2$ & $123(36.6 \%)$ \\
$3-4$ & $201(91.0 \%)$ \\
Child pugh & $20(9.0 \%)$ \\
A & $191(65.6 \%)$ \\
B & $100(34.4 \%)$ \\
Vascular invasion & \\
No & $48(32.2 \%)$ \\
Yes & $20(13.4 \%)$ \\
Etiology & $81(54.4 \%)$ \\
HBV & \\
HCV & \\
& \\
\hline & \\
\hline
\end{tabular}

The mRNA expression profile was transformed in RNA-Seq by Expectation Maximization (RSEM) values from the Illumina HiSeq 2000 RNA Sequencing platform and calculated as $\log _{2}(x+1)$ transformed RSEM normalized counts. A total of 341 patients in the TCGA dataset were included in the analysis. Patients with incomplete mRNA expression, OS, or recurrence-free survival (RFS) information were excluded. The median age of the patients was 61.0 years (range, 16 to 90 years). Other clinicopathological information is shown in Table 1. The median expression of FOXM1 and Aurora-A were 8.80 and 8.71, respectively. We used the cut-off finder online tool [12] (http://molpath.charite.de/cutoff/) to determine the optimal cut-off value of gene expression with prognostic significance. The cut-off values were 8.80 and 9.02 for FOXM1 and Aurora-A, respectively.

\subsection{Patient specimens}

A total of 40 diagnosed HCC specimens with adjacent non-cancerous tissues were used according to the ethical guidelines of the Declaration of Helsinki and was approved by the Institutional Review Board 
Table 2

Gene sets enrichment of FOXM1-related GO biological process gene sets in 341 HCC from TCGA dataset

\begin{tabular}{|c|c|c|c|c|c|}
\hline Gene sets & Size & ES & NES & NOM $p$-value & FDR $q$-value \\
\hline GO_CELL_CYCLE_PHASE_TRANSITION & 242 & 0.608 & 1.986 & 0.000 & 0.001 \\
\hline GO_DNA_REPAIR & 461 & 0.503 & 1.956 & 0.004 & 0.001 \\
\hline GO_SIGNAL_TRANSDUCTION_BY_P53_CLASS_MEDIATOR & 123 & 0.488 & 1.925 & 0.000 & 0.001 \\
\hline GO_CELL_CYCLE_G2_M_PHASE_TRANSITION & 132 & 0.552 & 1.917 & 0.000 & 0.001 \\
\hline GO_SIGNĀLTRANSSDUCTION_IN_RESPONSE_TO_DNA_DAMAGE & 95 & 0.518 & 1.887 & 0.000 & 0.001 \\
\hline GO_REGULATION_OF_CELL_CYCLE_ARREST & 107 & 0.504 & 1.830 & 0.000 & 0.004 \\
\hline GO_REGULATION_OF_CELL_AGING & 32 & 0.570 & 1.788 & 0.008 & 0.005 \\
\hline GO_NEGATIVE_REGULATION_OF_CELL_AGING & 16 & 0.631 & 1.646 & 0.021 & 0.021 \\
\hline
\end{tabular}

ES, enrichment score; NES, normalized enrichment score; NOM, nominal; FDR, false discovery rate.

(IRB) of Kaohsiung Medical University Hospital (KMUHIRB-F(I)-20170093). The median age of the patients was 60 years (range, 38 to 80 years). The follow-up period was from 45 to 2,402 days, with a median of 1,572 days.

\subsection{Gene set enrichment analysis (GSEA)}

For Gene Ontology (GO) biological process gene sets rank analysis, we used the Gene Set Expression Analysis (GSEA) tool $[13,14]$ to estimate the difference of FOXM1-related gene sets between all primary HCC and adjacent tissues. Each gene set would get an enrichment score (ES) that represented the degree of the overexpressed gene set. The nominal (NOM) $p$-value estimated the statistical significance of the enrichment score for a single gene set. The false discovery rate (FDR) was adjusted for gene set size, and FDR $<0.25$ were considered statistically significant.

\subsection{Quantitative real-time PCR (RT-qPCR)}

Total RNA was isolated from fresh-frozen tissues derived from HCC patients using Trizol reagent (Invitrogen) according to the manufacturer's instructions. Reverse transcription was performed using a HighCapacity cDNA Reverse Transcription Kit (Applied BioSystems). Diluted cDNA (10 ng) was used as a template, and the relative expression of each mRNA was evaluated using the StepOne Real-Time PCR Systems (Applied BioSystems). GAPDH mRNA was used as an internal control. The following primer sequences were used: for FOXM1, forward, 5'-ATACGTGGATTGAGG ACCACT-3', and reverse, 5' -TCCAATGTCAAGTAGC GGTTG-3'; Aurora-A, forward, 5'-GCTGGAGAGCTT AAAATTGCAG-3' and reverse, 5'-TTTTGTAGGTCT CTTGGTATGTG-3'; GAPDH, forward, 5'-AGCCACA TCGCTCAGACAC-3', and reverse, 5'-GCCCAATAC GACCAAATCC-3'

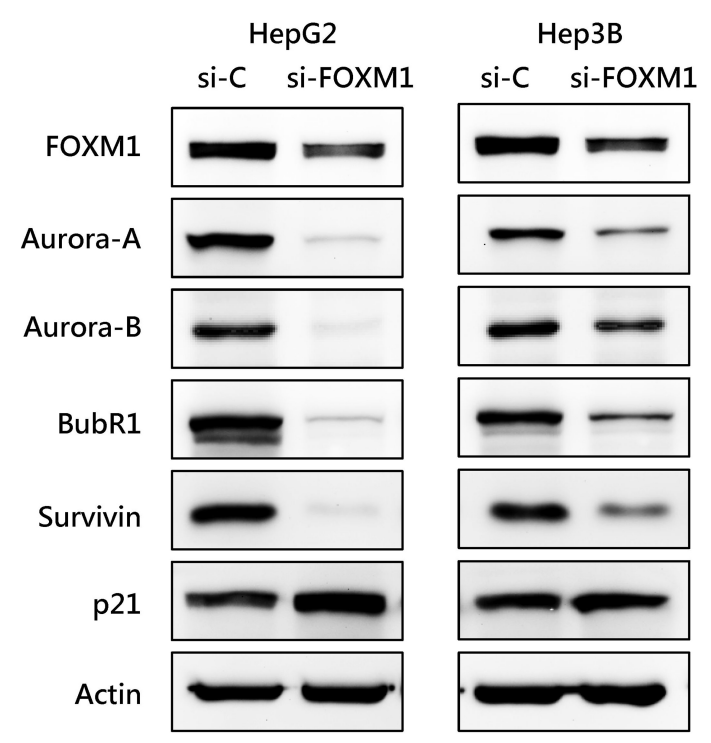

Supplementary Fig. 1. Effect of FOXM1 knockdown on the expression of cell cycle-regulating proteins in HCC cells. HepG2 and Hep3B cells were transfected with control siRNA or with FOXM1 siRNA. The expression of the indicated cell cycle-regulating proteins was analyzed by western blotting.

\section{5. siRNA and transfection}

The FOXM1 siRNA was purchased from Santa Cruz (sc-43769), and siRNAs were transfected into cells by jetPRIME $^{\circledR}$ (Polyplus transfection) according to the user instruction.

\subsection{Western blot analysis}

Western blot analysis was performed as we previously described [15]. The used antibodies are listed below. FOXM1 (sc-376471) and p21 (sc-6246) were purchased from Santa Cruz. Aurora-A (610404), Aurora-B (611083), BubR1 (612502) were purchased from BD Biosciences. Survivin (GTX100052) was purchased from GENETEX. Actin (A2228) was purchased from Sigma-Aldrich. 
(a)
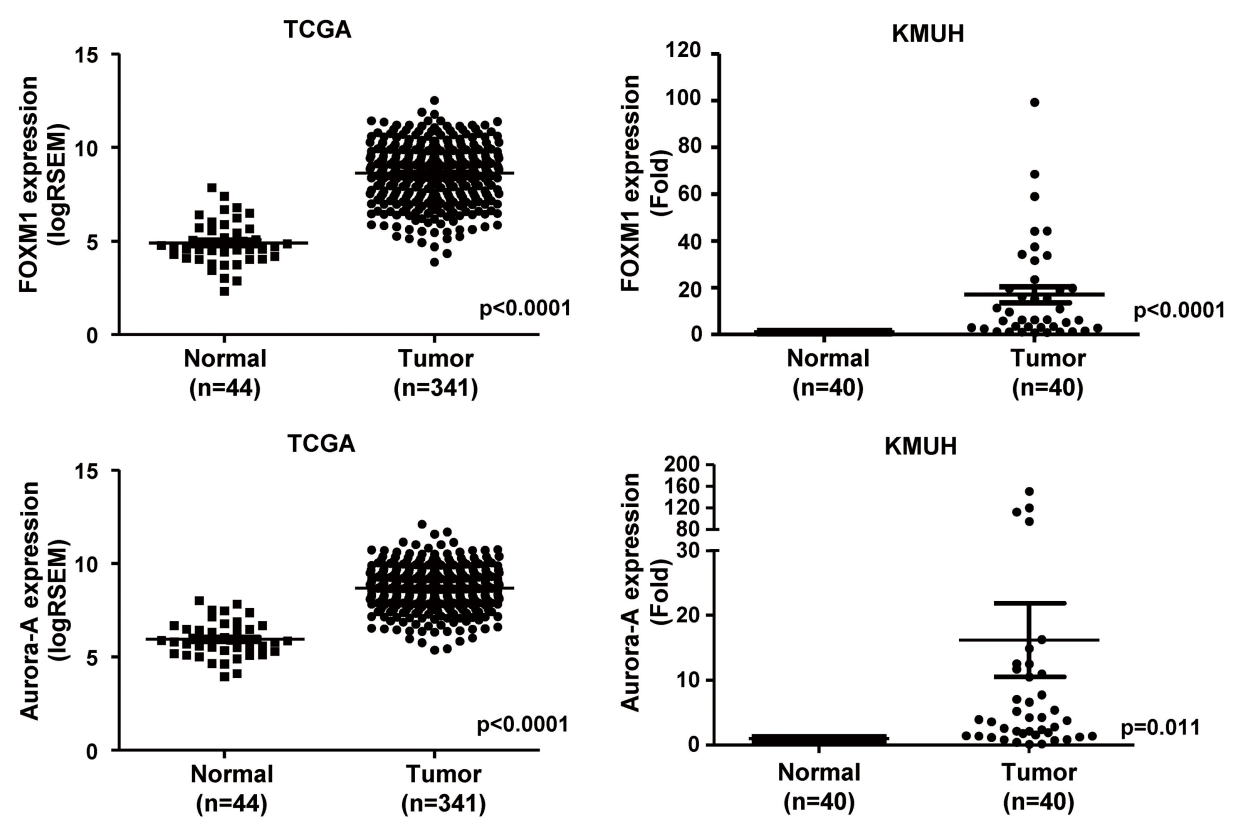

(b)

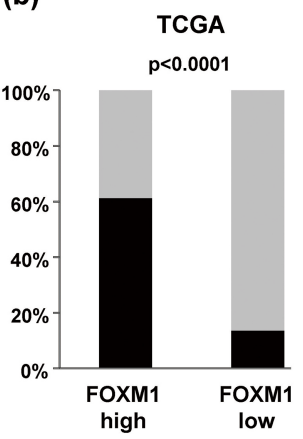

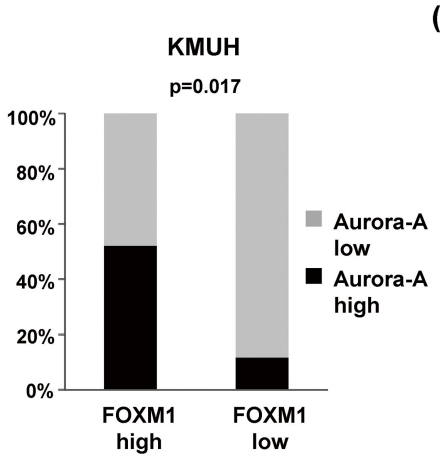

(c)

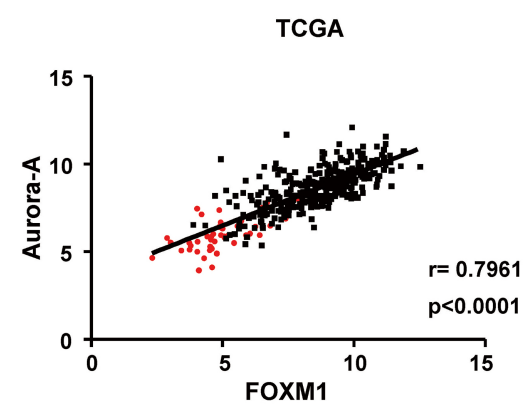

Fig. 1. FOXM1 expression is positively correlated with Aurora-A expression in human HCC. (a) The expression of FOXM1 and Aurora-A in HCC and adjacent liver tissues by transcriptome sequencing from the TCGA dataset (Left). The expression levels of the FOXM1 and Aurora-A mRNAs were validated in 40 paired HCC from KMUH using RT-qPCR (Right). The horizontal lines depict the mean \pm S.D. (b) The level of FOXM1 and Aurora-A in HCC tissues from the TCGA and KMUH datasets and the correlation between their expression levels was analyzed using Fisher's exact test $(p<0.001)$. (c) The relationship between FOXM1 and Aurora-A expression in HCC (black dot, $n=341$ ) and adjacent liver tissues (red dot, $n=44$ ) was analyzed using Pearson's correlation. RSEM, RNA-seq by Expectation Maximization.

\subsection{Statistical analysis}

All statistical analyses were performed using GraphPad Prism 5 (GraphPad Software, La Jolla, CA, USA) and SPSS 20.0 software (IBM, Chicago, USA). The two-tailed $t$-test was used to compare means between two groups. Pearson's chi-square test analyzed categorical data. Pearson's correlation coefficient was used to analyze the correlation between FOXM1 and Aurora-A. Survival curves were estimated by Kaplan-Meier and compared by the log-rank test. Cox regression analyses were conducted to assess the association between can- didate genes and survival. For all the tests, significance was accepted at $p<0.05$.

\section{Results}

\subsection{FOXM1 overexpression is correlated with Aurora-A upregulation in human $\mathrm{HCC}$}

We analyzed the expression pattern of FOXM1related pathways in HCC from the TCGA dataset using the Gene Set Enrichment Analysis (GSEA). A total of 
(a)

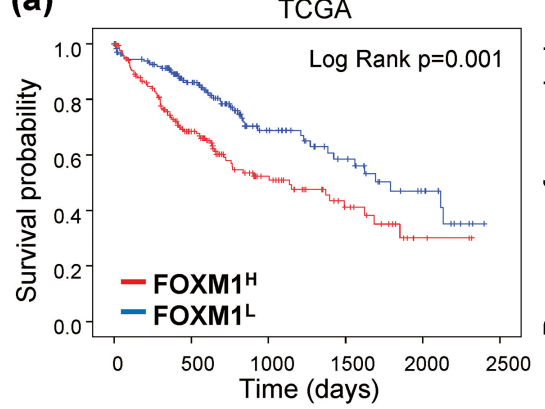

(b)

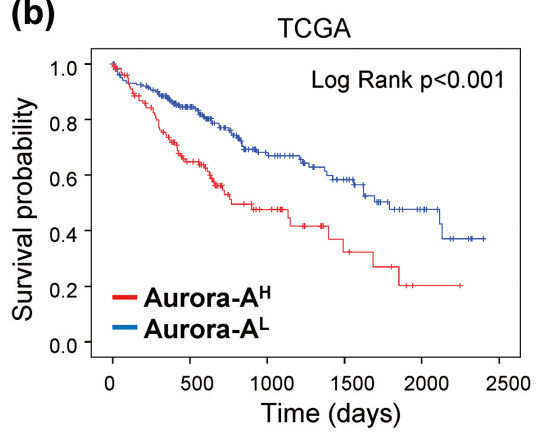

(e)

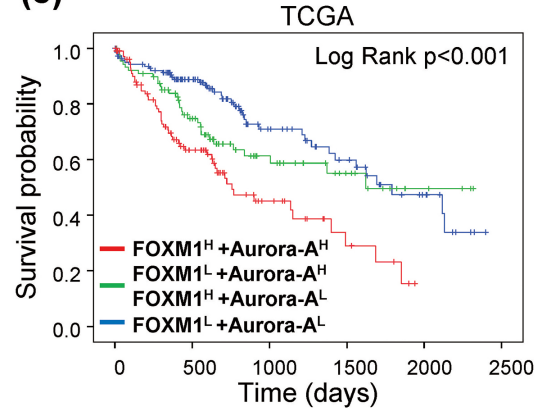

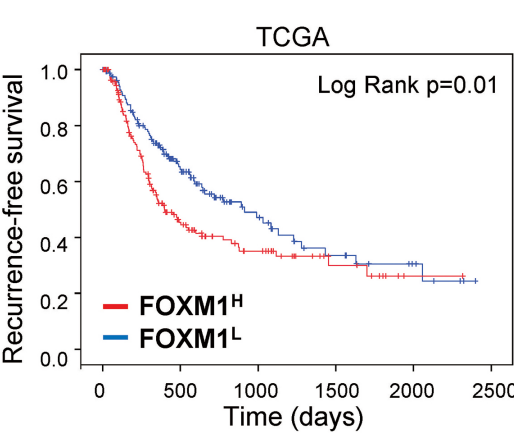

(c)

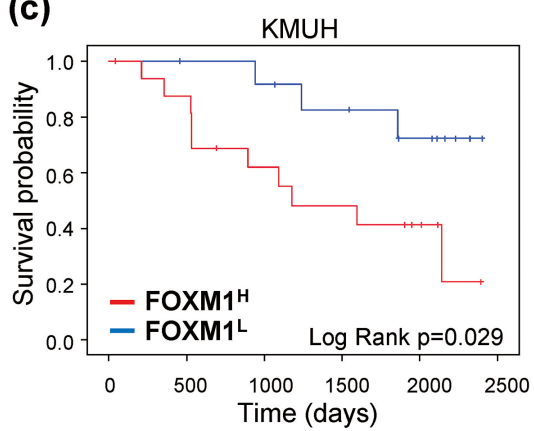

(d)
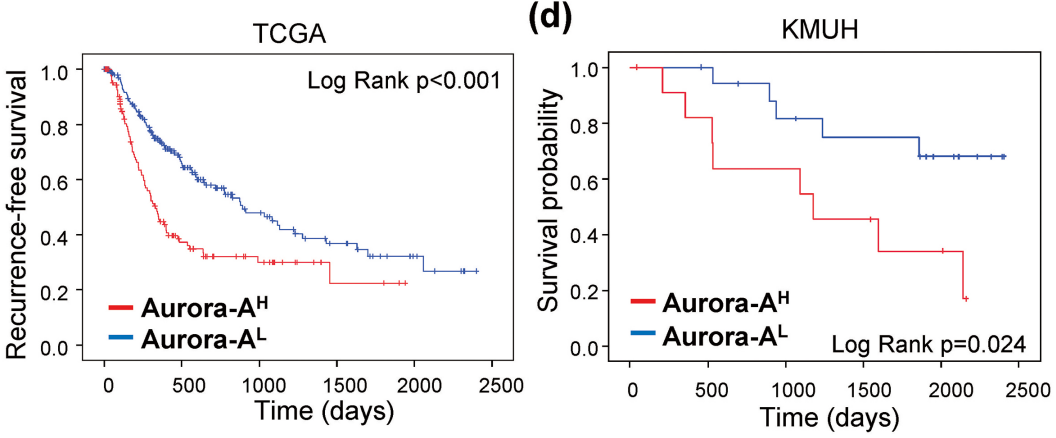

(f)

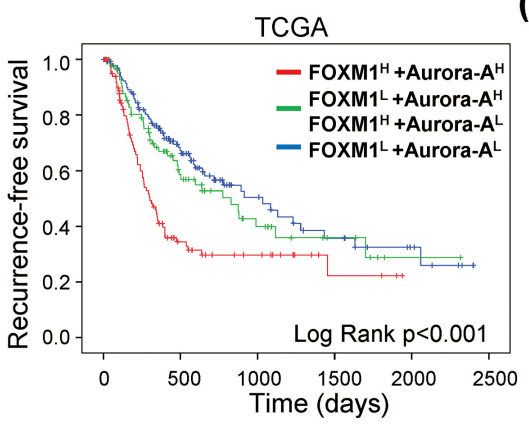

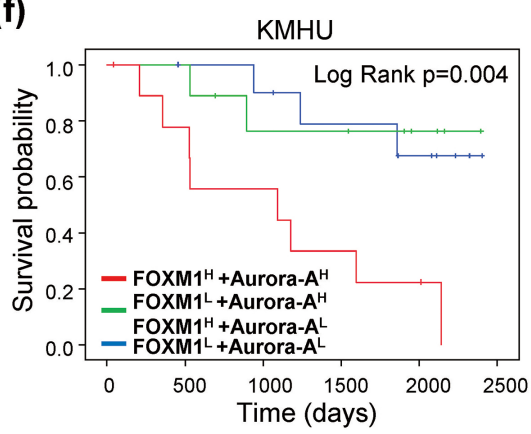

Fig. 2. Overexpression of FOXM1 and Aurora-A are associated with poor prognosis in HCC. KaplanMeier curves of OS (Left) and RFS (Right) were estimated with different FOXM1 and Aurora-A expression status in the TCGA $(n=341)(\mathrm{a}-\mathrm{b})$, and OS in KMUH ( $n=30)$ datasets (c-d). KaplanMeier curves for OS (Left) and RFS (Right) among three subgroups according to FOXM1 and Aurora-A expression status in the TCGA $(n=341)(\mathrm{e})$, and OS in KMUH $(n=30)$ datasets (f).

$341 \mathrm{HCC}$ and 44 normal tissues were included in the TCGA dataset. Eight FOXM1-related Gene Ontology (GO) biological process gene sets were significantly enriched in malignant HCC tissues (Table 2). Six of eight FOXM1-related gene sets were associated with cell cycle and DNA damage regulation. Knockdown of FOXM1 by siRNA decreased the expression of cell cycle-regulating proteins (Supplementary Fig. 1). This result showed that that FOXM1 is a critical cell cycle regulator. We found that Aurora-A (AURKA), a mitotic serine/threonine kinase, was involved in five FOXM1related gene sets and showed significantly high enrichment in HCC than normal tissues. Overexpression of
Aurora-A has been reported in a variety of solid tumors, including breast [16], colon [15], lung [17], and pancreas cancer [18]. The deregulation of Aurora-A may result in chromosomal instability and thus be related to tumorigenesis. Furthermore, Aurora-A has been identified as a target of FOXM1 in melanoma [19] and breast cancer [20]. Thus, we speculate that the level of FOXM1 may be increased in parallel with Aurora-A expression. We found that the relative expression of both the FOXM1 and Aurora-A mRNAs were significantly higher in HCC tissues than in adjacent liver tissues from the TCGA RNA-seq dataset. Consistently, the results of RT-qPCR indicated that the expression of 
Table 3

The correlation between clinical variables and FOXM1 and Aurora-A expression in HCC patients obtained from the TCGA dataset

\begin{tabular}{|c|c|c|c|c|}
\hline Clinical variables & $\begin{array}{c}\mathrm{F}^{\mathrm{L}}+\mathrm{A}^{\mathrm{L}} \\
N=145(42.5 \%)\end{array}$ & $\begin{array}{c}\mathrm{F}^{\mathrm{L}}+\mathrm{A}^{\mathrm{H}} / \mathrm{F}^{\mathrm{H}}+\mathrm{A}^{\mathrm{L}} \\
N=90(26.4 \%)\end{array}$ & $\begin{array}{c}\mathrm{F}^{\mathrm{H}}+\mathrm{A}^{\mathrm{H}} \\
N=106(31.1 \%)\end{array}$ & $p$-value ${ }^{\mathrm{a}}$ \\
\hline Age & & & & 0.113 \\
\hline$<60$ & $56(37.1 \%)$ & $40(26.5 \%)$ & $55(36.4 \%)$ & \\
\hline$\geqslant 60$ & $89(46.8 \%)$ & $50(26.3 \%)$ & $51(26.8 \%)$ & \\
\hline Gender & & & & 0.707 \\
\hline Male & $101(43.7 \%)$ & $58(25.1 \%)$ & $72(31.2 \%)$ & \\
\hline Female & $44(40.0 \%)$ & $32(29.1 \%)$ & $34(30.9 \%)$ & \\
\hline \multicolumn{5}{|l|}{ TNM stage } \\
\hline $\mathrm{T} 1-2$ & $117(46.2 \%)$ & $66(26.1 \%)$ & $70(27.7 \%)$ & 0.024 \\
\hline T3-4 & $27(31.4 \%)$ & $23(26.7 \%)$ & $36(41.9 \%)$ & \\
\hline No & $88(38.1 \%)$ & $67(29.0 \%)$ & $76(32.9 \%)$ & 0.886 \\
\hline N1 & $2(50.0 \%)$ & $1(25.0 \%)$ & $1(25.0 \%)$ & \\
\hline M0 & $99(40.4 \%)$ & $60(24.5 \%)$ & $86(35.1 \%)$ & 0.438 \\
\hline M1 & $2(66.7 \%)$ & $1(33.3 \%)$ & $0(0.0 \%)$ & \\
\hline Stage & & & & 0.031 \\
\hline $1-2$ & $110(46.4 \%)$ & $62(26.2 \%)$ & $65(27.4 \%)$ & \\
\hline $3-4$ & $26(31.7 \%)$ & $22(26.8 \%)$ & $34(41.5 \%)$ & \\
\hline Grade & & & & $<0.001$ \\
\hline $1-2$ & $110(51.6 \%)$ & $53(24.9 \%)$ & $50(23.5 \%)$ & \\
\hline $3-4$ & $33(26.8 \%)$ & $36(29.3 \%)$ & $54(43.9 \%)$ & \\
\hline Child pugh & & & & 0.027 \\
\hline $\mathrm{A}$ & $90(44.8 \%)$ & $55(27.4 \%)$ & $56(27.9 \%)$ & \\
\hline B & $7(35.0 \%)$ & $11(55.0 \%)$ & $2(10.0 \%)$ & \\
\hline Vascular invasion & & & & 0.594 \\
\hline No & $88(46.1 \%)$ & $56(29.3 \%)$ & $47(24.6 \%)$ & \\
\hline Yes & $44(44.0 \%)$ & $26(26.0 \%)$ & $30(30.0 \%)$ & \\
\hline Etiology & & & & 0.003 \\
\hline $\mathrm{HBV}$ & $18(37.5 \%)$ & $18(37.5 \%)$ & $12(25.0 \%)$ & \\
\hline $\mathrm{HCV}$ & $8(40.0 \%)$ & $10(50.0 \%)$ & $2(10.0 \%)$ & \\
\hline $\mathrm{HBV}+\mathrm{HCV}$ & $26(32.1 \%)$ & $16(19.8 \%)$ & $39(48.1 \%)$ & \\
\hline
\end{tabular}

aThe data were analyzed using Pearson's chi-square test. $\mathrm{F}^{\mathrm{H}}$, high FOXM1; $\mathrm{F}^{\mathrm{L}}$, low FOXM1; $\mathrm{A}^{\mathrm{H}}$, high Aurora-A; $\mathrm{A}^{\mathrm{L}}$, low Aurora-A.

FOXM1 and Aurora-A mRNAs were highly expressed in HCC tissues in the KMUH dataset (Fig. 1a). It was showed that $50.7 \%(173 / 341)$ and $37.8 \%(129 / 341)$ of patients with high FOXM1 and Aurora-A expression tissues from the TCGA dataset, respectively. The proportion of high FOXM1 and Aurora-A expression tissues in the KMUH dataset was $57.5 \%(23 / 40)$ and $35.0 \%$ (14/40), respectively. High Aurora-A expression was observed more frequently detected in high FOXM1 than in low FOXM1 tissues (61.3\% vs. $13.7 \%$, TCGA; $52.2 \%$ vs. $11.8 \%$, KMUH) (Fig. 1 b). We also found a positive linear correlation between FOXM1 and Aurora-A expression in HCC tissues $(r=0.7961$, $P<0.0001$ ) (Fig. 1c). Overall, these results indicate that both FOXM1 and Aurora-A were parallel overexpressed in HCC.

\subsection{Coordinated overexpression of FOXM1 and} Aurora-A are associated with poor prognosis of HCC patients

To investigate the prognostic values of FOXM1 and
Aurora-A expression in HCC, we separated the HCC patients into low- and high-expression groups. $\mathrm{Pa}-$ tients with high FOXM1 expression $\left(\mathrm{FOXM} 1^{\mathrm{H}}\right)$ or high Aurora-A expression (Aurora- $\mathrm{A}^{\mathrm{H}}$ ) had significantly shorter OS and RFS than that with low FOXM1 expression $\left(\mathrm{FOXM} 1^{\mathrm{L}}\right.$ ) or low Aurora-A expression (Aurora$\mathrm{A}^{\mathrm{L}}$ ) in TCGA datasets (Fig. 2a and b). In the KMUH cohort, patients with FOXM1 ${ }^{\mathrm{H}}$ or Aurora- $\mathrm{A}^{\mathrm{H}}$ also exhibited poor OS (Fig. 2c and d). We further classified the patients into three groups according to the FOXM1 and Aurora-A expression: both high-expression $\left(\mathrm{F}^{\mathrm{H}}+\right.$ $\left.\mathrm{A}^{\mathrm{H}}\right)$, both low-expression $\left(\mathrm{F}^{\mathrm{L}}+\mathrm{A}^{\mathrm{L}}\right)$, and intermediate $\left(\mathrm{F}^{\mathrm{H}}+\mathrm{A}^{\mathrm{L}} / \mathrm{F}^{\mathrm{L}}+\mathrm{A}^{\mathrm{H}}\right)$ groups. We found that the fiveyear OS rates for the TCGA dataset were $23 \%$ in the $\mathrm{F}^{\mathrm{H}}+\mathrm{A}^{\mathrm{H}}$ group, $50 \%$ in the $\mathrm{F}^{\mathrm{H}}+\mathrm{A}^{\mathrm{L}} / \mathrm{F}^{\mathrm{L}}+\mathrm{A}^{\mathrm{H}}$ group, and $47 \%$ in the $\mathrm{F}^{\mathrm{L}}+\mathrm{A}^{\mathrm{L}}$ group $(\log$-rank $p<0.001)$ (Fig. 2e). Five-year OS rates for the three groups in KMUH dataset were $22 \%, 76 \%$, and $79 \%$, respectively (log-rank $p=0.004$ ) (Fig. 2f). Five-year RFS was $22 \%$ in $\mathrm{F}^{\mathrm{H}}+\mathrm{A}^{\mathrm{H}}$ group, compared with $29 \%$ and $32 \%$ in $\mathrm{F}^{\mathrm{H}}+\mathrm{A}^{\mathrm{L}} / \mathrm{F}^{\mathrm{L}}+\mathrm{A}^{\mathrm{H}}$ and $\mathrm{F}^{\mathrm{L}}+\mathrm{A}^{\mathrm{L}}$ group, respectively 
Table 4

Univariate and multivariate Cox regression analysis of variables associated with overall survival and recurrence-free survival in HCC from the TCGA dataset

\begin{tabular}{|c|c|c|c|c|}
\hline \multirow[t]{2}{*}{ Variables } & \multicolumn{2}{|l|}{ Univariate } & \multicolumn{2}{|l|}{ Multivariate } \\
\hline & HR $(95 \% \mathrm{CI})$ & $p$ value $^{\mathrm{a}}$ & HR $(95 \% \mathrm{CI})$ & $p$-value \\
\hline \multicolumn{5}{|l|}{ Overall survival } \\
\hline Age $(\geqslant 60$ vs. $<60)$ & $1.168(0.812-1.680)$ & 0.404 & & \\
\hline Gender (Male vs. Female) & $1.343(0.931-1.937)$ & 0.115 & & \\
\hline T (T3-T4 vs. T1-T2) & $2.624(1.823-3.778)$ & $<\mathbf{0 . 0 0 1}$ & $1.182(0.158-8.841)$ & 0.871 \\
\hline N (N1 vs. N0) & $1.821(0.446-7.440)$ & 0.404 & & \\
\hline M (M1 vs. M0) & $3.648(1.146-11.609)$ & 0.028 & $2.946(0.875-9.925)$ & 0.081 \\
\hline Stage (3-4 vs. $1-2)$ & $2.515(1.714-3.692)$ & $<\mathbf{0 . 0 0 1}$ & $2.307(0.311-17.125)$ & 0.414 \\
\hline Grade (3-4 vs. $1-2)$ & $1.096(0.756-1.589)$ & 0.629 & & \\
\hline Child pugh (B vs. A) & $1.854(0.878-3.915)$ & 0.105 & & \\
\hline Vascular invasion (Yes vs. No) & $1.398(0.909-2.149)$ & 0.127 & & \\
\hline FOXM1 (High vs. Low) & $1.858(1.287-2.682)$ & 0.001 & & \\
\hline Aurora-A (High vs. Low) & $2.042(1.423-2.932)$ & $<0.001$ & & \\
\hline \multicolumn{5}{|l|}{ FOXM1/Aurora-A } \\
\hline $\mathrm{F}^{\mathrm{H}}+\mathrm{A}^{\mathrm{L}} / \mathrm{F}^{\mathrm{L}}+\mathrm{A}^{\mathrm{H}}$ vs. $\mathrm{F}^{\mathrm{L}}+\mathrm{A}^{\mathrm{L}}$ & $1.405(0.880-2.244)$ & 0.154 & & \\
\hline $\mathrm{F}^{\mathrm{H}}+\mathrm{A}^{\mathrm{H}}$ vs. $\mathrm{F}^{\mathrm{L}}+\mathrm{A}^{\mathrm{L}}$ & $2.497(1.634-3.816)$ & $<\mathbf{0 . 0 0 1}$ & $3.065(1.771-5.307)$ & $<0.001$ \\
\hline \multicolumn{5}{|l|}{ Recurrence-free survival } \\
\hline Age $(\geqslant 60$ vs. $<60)$ & $1.027(0.752-1.404)$ & 0.865 & & \\
\hline Gender (Male vs. Female) & $1.017(0.729-1.419)$ & 0.920 & & \\
\hline T (T3-T4 vs. T1-T2) & $2.381(1.713-3.309)$ & $<\mathbf{0 . 0 0 1}$ & $0.354(0.046-2.727)$ & 0.319 \\
\hline N (N1 vs. N0) & $1.395(0.344-5.661)$ & 0.641 & & \\
\hline M (M1 vs. M0) & $2.379(0.582-9.732)$ & 0.228 & & \\
\hline Stage (3-4 vs. 1-2) & $2.369(1.681-3.337)$ & $<\mathbf{0 . 0 0 1}$ & $4.772(0.642-35.476)$ & 0.127 \\
\hline Grade (3-4 vs. $1-2)$ & $1.111(0.805-1.533)$ & 0.523 & & \\
\hline Child pugh (B vs. A) & $1.265(0.634-2.524)$ & 0.504 & & \\
\hline Vascular invasion (Yes vs. No) & $1.910(1.332-2.738)$ & $<\mathbf{0 . 0 0 1}$ & $1.595(1.075-2.367)$ & 0.020 \\
\hline FOXM1 (High vs. Low) & $1.502(1.099-2.054)$ & 0.011 & & \\
\hline Aurora-A (High vs. Low) & $1.961(1.434-2.683)$ & $<0.001$ & & \\
\hline \multicolumn{5}{|l|}{ FOXM1/Aurora-A } \\
\hline $\mathrm{F}^{\mathrm{L}}+\mathrm{A}^{\mathrm{H}} / \mathrm{F}^{\mathrm{H}}+\mathrm{A}^{\mathrm{L}}$ vs. $\mathrm{F}^{\mathrm{L}}+\mathrm{A}^{\mathrm{L}}$ & $1.206(0.808-1.800)$ & 0.359 & & \\
\hline $\mathrm{F}^{\mathrm{H}}+\mathrm{A}^{\mathrm{H}}$ vs. $\mathrm{F}^{\mathrm{L}}+\mathrm{A}^{\mathrm{L}}$ & $2.111(1.469-3.032)$ & $<0.001$ & $1.749(1.126-2.717)$ & 0.013 \\
\hline
\end{tabular}

${ }^{\mathrm{a}} p$-value given in bold was used to adjust for Multivariate analysis. $\mathrm{F}^{\mathrm{H}}$, high FOXM1; $\mathrm{F}^{\mathrm{L}}$, low FOXM1; $\mathrm{A}^{\mathrm{H}}$, high Aurora-A; $\mathrm{A}^{\mathrm{L}}$, low Aurora-A.

(log-rank $p<0.001$ ) (Fig. 2e). From the results, patients with both FOXM1 and Aurora-A high-expression had significantly shorter overall survival and earlier recurrence.

Moreover, $\mathrm{F}^{\mathrm{H}}+\mathrm{A}^{\mathrm{H}}$ expression was positively correlated with larger tumor size $(p=0.024)$, advanced stage $(p=0.031)$, higher grade $(p<0.001)$, and doublepositive for HBV and HCV $(p=0.003)$. However, patients with $\mathrm{F}^{\mathrm{H}}+\mathrm{A}^{\mathrm{H}}$ expression had high proportion of Child pugh A class ( $p=0.027$ ) (Table 3 ).

We further confirm the association of FOXM1 and Aurora-A expression with OS and RFS by Cox regression analysis. Univariate Cox regression analysis demonstrated that $\mathrm{T}$ classification, $\mathrm{M}$ classification, stage, and FOXM1 and/or Aurora-A expression were all significantly associated with OS. T classification, stage, vascular invasion, and FOXM1 and/or Aurora-A expression were correlated with RFS. Subsequently, the significant characteristics factors from the univariate analyses were included for multivariate Cox regression analysis. The results showed that $\mathrm{F}^{\mathrm{H}}+\mathrm{A}^{\mathrm{H}}$ expression was the most significant independent predictor for OS (HR: $3.065, p<0.001$ ) and was for RFS (HR: 1.749, $p=0.013$ ), and vascular invasion was also an independent prognostic factor for RFS (Table 4).

\subsection{Co-expression of FOXM1 and Aurora-A predicts the prognosis of sorafenib-treated patients in $\mathrm{HCC}$}

In a subsequent analysis, we found that the $\mathrm{F}^{\mathrm{H}}+\mathrm{A}^{\mathrm{H}}$ expression in 29 sorafenib-treated patients (TCGA cohort) was associated with worse survival in HCC. The five-year OS rates was significantly worse in FOXM1 ${ }^{\mathrm{H}}$ patients compared with FOXM1 ${ }^{\mathrm{L}}$ patients $(15 \%$ vs. $67 \%, p=0.03$ ) (Fig. 3a). A significant difference in five-year OS rates was also seen in Aurora- $\mathrm{A}^{\mathrm{H}}$ between Aurora- $\mathrm{A}^{\mathrm{L}}$ patients (0\% vs. $40 \%, p=0.029$ ) (Fig. 3a). The five-year OS rates was zero for $\mathrm{F}^{\mathrm{H}}+\mathrm{A}^{\mathrm{H}}$ patients, compared with $28 \%$ and $67 \%$ in $\mathrm{F}^{\mathrm{H}}+\mathrm{A}^{\mathrm{L}} / \mathrm{F}^{\mathrm{L}}+\mathrm{A}^{\mathrm{H}}$ and 
(a)

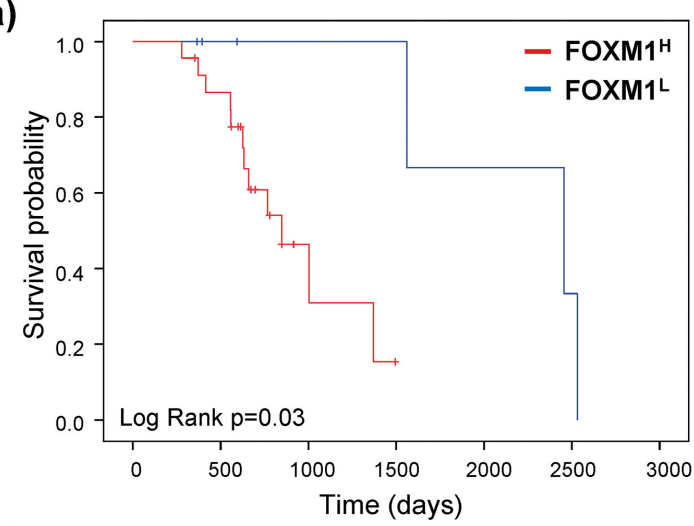

(b)

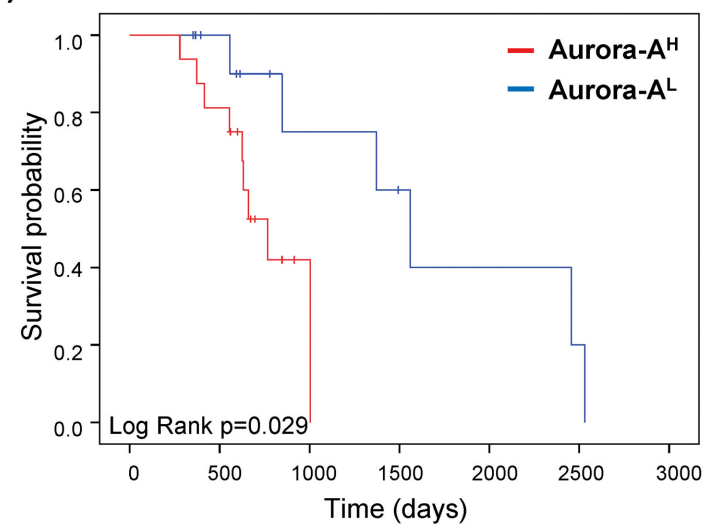

(c)

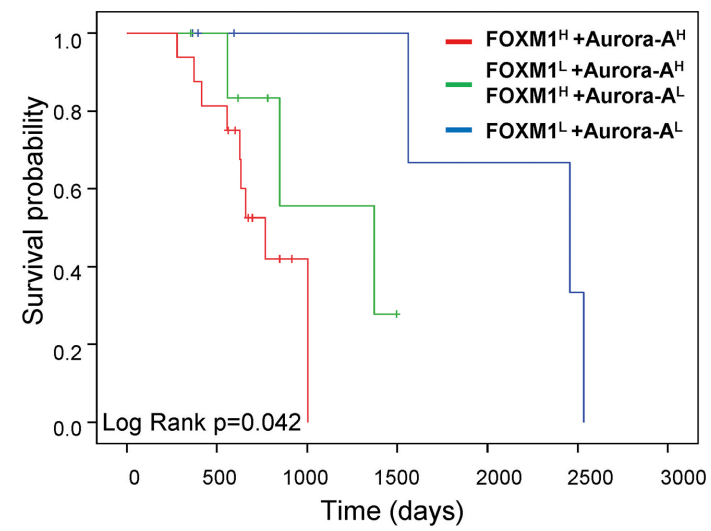

Fig. 3. FOXM1 and Aurora-A expression predict the prognosis of sorafenib-treated patients in HCC. KaplanMeier curves of overall survival probability were estimated with different FOXM1 (a), Aurora-A (b), and FOXM1/Aurora-A (c) expression status in sorafenib-treated patients obtained from the TCGA dataset $(n=29)$.

$\mathrm{F}^{\mathrm{L}}+\mathrm{A}^{\mathrm{L}}$ patients, respectively $(p=0.042)$ (Fig. 3c). These results suggest that FOXM1 and Aurora-A expression are key factors associated with a poor prognosis in sorafenib-treated HCC patients, and they may be effective in predicting the efficacy of sorafenib.

\section{Discussion}

Valid and precise biomarkers for identifying patients who have a poor prognosis are urgently needed to optimize their individual therapy. The development of powerful target-therapeutic drugs for $\mathrm{HCC}$ is also critical for this aggressive cancer. FOXM1 is a critical regulator in the G1/S and G2/M transitions of the cell cycle through regulating downstream targets such as centromere protein (CENP) F, cyclin B1, kinesin family member (KIF), p $27^{\text {Kip } 1}$ and p $21^{\text {cip } 1}$ in HCC cells $[21,22]$. Our GSEA data also showed that FOXM1-related gene sets were mostly associated with cell cycle regulation in HCC (Table 2). We suppose that the abnormal expression of FOXM1 may contribute to deregulating cell cycle and induce cell proliferation in HCC. Our finding revealed that FOXM1 overexpression is positively correlated with a vital cell cycle regulator, Aurora- $\mathrm{A}$, in human HCC (Fig. 1c). The proportion of FOXM $1^{\mathrm{H}}$ and Aurora- $\mathrm{A}^{\mathrm{H}}$ tissues was as many as $50 \%$ and $35 \%$ in both TCGA and KMUH cohort, respectively. It is noteworthy that FOXM $1^{\mathrm{H}}$ and Aurora- $\mathrm{A}^{\mathrm{H}}$ expression were more associated with the Asian race than the White race (data not shown). Moreover, it was demonstrated that FOXM1 could predict the prognosis of HCC in two Asian cohorts, and Aurora-A was a critical hub gene with prognostic value using the bioinformatics analysis $[7,23,24]$. From the literature review, we hypothesized that the expression of FOXM1 and Aurora-A could be prognostic and predictive biomarkers for HCC.

In this study, we determined the relationship of FOXM1 and Aurora-A expression and analyzed the significance of their expression on the prognosis of HCC. There was $31.1 \%(106 / 341)$ of patients with $\mathrm{F}^{\mathrm{H}}+\mathrm{A}^{\mathrm{H}}$ expression and they were associated with larger tumor size, advanced stage, higher-grade tumors, and poorer outcome (Table 3 and Fig. 2). A meta-analysis demonstrated that FOXM $1^{\mathrm{H}}$ expression could predict worse prognosis of $\mathrm{HCC}$ with advanced vascular invasion, differentiation, and TNM stage [25]. These results suggested that FOXM1 and Aurora-A cloud cooperatively play essential roles in inducing tumor proliferation and progression.

$\mathrm{F}^{\mathrm{H}}+\mathrm{A}^{\mathrm{H}}$ expression was positively correlated with double-positive for HBV and HCV (Table 3). It seems that virus infection could be a key factor in affecting the expression of FOXM1 or Aurora-A. It is consistent with a previous report that $\mathrm{HBx}$ can upregulate FOXM1 expression to promote tumor metastasis and poor prognosis [26], and $\mathrm{HCV}$ can increase the expression Aurora-A in HCC tissues [27]. However, there are 
still no reports showing synergetic effects of coexisting HBV and HCV to FOXM1 or Aurora-A expression.

In univariate Cox regression analysis, $\mathrm{F}^{\mathrm{H}}+\mathrm{A}^{\mathrm{H}}$ expression (HR: 2.497, $p<0.001$ ) was the most significant factor associated with OS than FOXM1 (HR: $1.858, p=0.001$ ) or Aurora-A (HR: 2.042, $p<0.001$ ) alone. $\mathrm{F}^{\mathrm{H}}+\mathrm{A}^{\mathrm{H}}$ expression (HR: 2.111, $p<0.001$ ) enhanced the prognostic value for RFS than FOXM1 alone (HR: $1.502, p=0.011$ ). Although some studies have identified FOXM1 and Aurora-A differentially expressed, respectively, in $\mathrm{HCC}$, their combinational predictive value has not been specifically investigated. So far, this is the first study to propose that the coexpression of FOXM1 and Aurora-A can be a new prognostic biomarker for HCC patients.

Unlike other malignancies, such as breast [28] and lung cancer [29], which have reliable biomarkers for treatment decisions in clinical practice, there is no biomarker for selecting HCC patients who could benefit most from sorafenib. Previous studies showed that FOXM1 and Aurora-A are related to TKI resistance in lung cancer $[11,30]$. The level of FOXM1 was correlated with sorafenib resistance in HCC cells. High FOXM1 expression was found in sorafenib-resistant cells, whereas low levels were observed in the comparatively sorafenib-sensitive cells [31,32]. Furthermore, Aurora-A has been suggested to be a critical therapeutic target combine with sorafenib to improve the HCC patient's drug resistance and prolong survival [33]. According to the above experimental results, we suppose that different levels of FOXM1 and Aurora-A react differently when exposed to sorafenib. In our pilot analysis of $\mathrm{HCC}$ specimens prove that the $\mathrm{F}^{\mathrm{H}}+\mathrm{A}^{\mathrm{H}}$ expression in sorafenib-treated patients (TCGA cohort) was associated with worse survival in HCC (Fig. 3), suggesting that the FOXM and Aurora-A expression could affect the therapeutic efficiency of sorafenib. Thus, FOXM1 and Aurora-A could be a reliable biomarker to predict drug response and prognosis of HCC patients. It is also reasonable to propose that adding FOXM1 or Aurora-A inhibitor to sorafenib may enhance the cytotoxic effects of sorafenib in HCC.

In conclusion, we found that the expression of FOXM1 was positively associated with the levels of Aurora-A in HCC. $\mathrm{F}^{\mathrm{H}}+\mathrm{A}^{\mathrm{H}}$ expression was associated with poor prognosis and was the most significant independent prognostic factor for OS and RFS. Moreover, $\mathrm{F}^{\mathrm{H}}+\mathrm{A}^{\mathrm{H}}$ expression was a key factor associated with a poor prognosis of sorafenib-treated HCC patients, and it was effective in predicting sorafenib response, suggesting that the co-expression of FOXM1 and Aurora-A could be a reliable biomarker to predict sorafenib response and prognosis of HCC patients. Both FOXM1 and Aurora-A are potential therapeutic targets for HCC.

\section{Acknowledgments}

This work was supported by MOST grants (MOST 106-2320-B-037-001-MY3; MOST 107-2314-B-037026) and KMUH grant (KMUH105-5R29).

\section{Conflict of interest}

The authors declare that they have no conflicts of interest.

\section{References}

[1] F. Bray, J. Ferlay, I. Soerjomataram, R.L. Siegel, L.A. Torre and A. Jemal, Global cancer statistics 2018: GLOBOCAN estimates of incidence and mortality worldwide for 36 cancers in 185 countries, CA Cancer J Clin 68 (2018), 394-424.

[2] D. Dimitroulis, C. Damaskos, S. Valsami, S. Davakis, N. Garmpis, E. Spartalis, A. Athanasiou, D. Moris, S. Sakellariou, S. Kykalos, G. Tsourouflis, A. Garmpi, I. Delladetsima, K. Kontzoglou and G. Kouraklis, From diagnosis to treatment of hepatocellular carcinoma: An epidemic problem for both developed and developing world, World J Gastroenterol $\mathbf{2 3}$ (2017), 5282-5294.

[3] P.J. Johnson, The role of serum alpha-fetoprotein estimation in the diagnosis and management of hepatocellular carcinoma, Clin Liver Dis 5 (2001), 145-159.

[4] E.S. Bialecki and A.M. Di Bisceglie, Diagnosis of hepatocellular carcinoma, HPB (Oxford) 7 (2005), 26-34.

[5] G.B. Liao, X.Z. Li, S. Zeng, C. Liu, S.M. Yang, L. Yang, C.J. $\mathrm{Hu}$ and J.Y. Bai, Regulation of the master regulator FOXM1 in cancer, Cell Commun Signal 16 (2018), 57.

[6] J. Wang, Y. Tian, H. Chen, H. Li and S. Zheng, Key signaling pathways, genes and transcription factors associated with hepatocellular carcinoma, Mol Med Rep 17 (2018), 8153-8160.

[7] B.N. Song and I.S. Chu, A gene expression signature of FOXM1 predicts the prognosis of hepatocellular carcinoma, Exp Mol Med 50 (2018), e418.

[8] P. Laissue, The forkhead-box family of transcription factors: key molecular players in colorectal cancer pathogenesis, $\mathrm{Mol}$ Cancer 18 (2019), 5.

[9] L. Li, D. Wu, Q. Yu, L. Li and P. Wu, Prognostic value of FOXM1 in solid tumors: a systematic review and metaanalysis, Oncotarget 8 (2017), 32298-32308.

[10] S. Abdeljaoued, L. Bettaieb, M. Nasri, O. Adouni, A. Goucha, H. Bouzaiene, H. Boussen, K. Rahal and A. Gamoudi, Forkhead box M1 (FOXM1) expression predicts disease free survival and may mediate resistance to chemotherapy and hormonotherapy in male breast cancer, Breast Dis 37 (2018), 109-114.

[11] M. Li, J. Yang, W. Zhou, Y. Ren, X. Wang, H. Chen, J. Zhang, J. Chen, Y. Sun, L. Cui, X. Liu, L. Wang and C. Wu, Activa- 
tion of an AKT/FOXM1/STMN1 pathway drives resistance to tyrosine kinase inhibitors in lung cancer, Br J Cancer 117 (2017), 974-983.

[12] J. Budczies, F. Klauschen, B.V. Sinn, B. Gyorffy, W.D. Schmitt, S. Darb-Esfahani and C. Denkert, Cutoff finder: A comprehensive and straightforward Web application enabling rapid biomarker cutoff optimization, PLoS One 7 (2012), e51862.

[13] A. Subramanian, P. Tamayo, V.K. Mootha, S. Mukherjee, B.L Ebert, M.A. Gillette, A. Paulovich, S.L. Pomeroy, T.R. Golub, E.S. Lander and J.P. Mesirov, Gene set enrichment analysis: A knowledge-based approach for interpreting genome-wide expression profiles, Proc Natl Acad Sci U S A 102 (2005), 15545-15550.

[14] V.K. Mootha, C.M. Lindgren, K.F. Eriksson, A. Subramanian, S. Sihag, J. Lehar, P. Puigserver, E. Carlsson, M. Ridderstrale, E. Laurila, N. Houstis, M.J. Daly, N. Patterson, J.P. Mesirov, T.R. Golub, P. Tamayo, B. Spiegelman, E.S. Lander, J.N. Hirschhorn, D. Altshuler and L.C. Groop, PGC-1alpharesponsive genes involved in oxidative phosphorylation are coordinately downregulated in human diabetes, Nat Genet 34 (2003), 267-273.

[15] Y.C. Wang, K.C. Chang, B.W. Lin, J.C. Lee, C.H. Lai, L.J. Lin, Y. Yen, C.S. Lin, S.J. Yang, P.C. Lin, C.T. Lee and L.Y. Hung, The EGF/hnRNP Q1 axis is involved in tumorigenesis via the regulation of cell cycle-related genes, Exp Mol Med $\mathbf{5 0}$ (2018), 70 .

[16] S. Jiang, H. Katayama, J. Wang, S.A. Li, Y. Hong, L. Radvanyi, J.J. Li and S. Sen, Estrogen-induced aurora kinase-A (AURKA) gene expression is activated by GATA-3 in estrogen receptor-positive breast cancer cells, Horm Cancer 1 (2010), $11-20$.

[17] E. Ogawa, K. Takenaka, H. Katakura, M. Adachi, Y. Otake, Y Toda, H. Kotani, T. Manabe, H. Wada and F. Tanaka, Perimembrane Aurora-A expression is a significant prognostic factor in correlation with proliferative activity in non-small-cell lung cancer (NSCLC), Ann Surg Oncol 15 (2008), 547-554.

[18] J. Wang, K. Nikhil, K. Viccaro, L. Chang, M. Jacobsen, G. Sandusky and K. Shah, The Aurora-A-Twist1 axis promotes highly aggressive phenotypes in pancreatic carcinoma, $\mathrm{J}$ Cell Sci 130 (2017), 1078-1093.

[19] J.A. Puig-Butille, A. Vinyals, J.R. Ferreres, P. Aguilera, E. Cabre, G. Tell-Marti, J. Marcoval, F. Mateo, L. Palomero, C. Badenas, J.M. Piulats, J. Malvehy, M.A. Pujana, S. Puig and A. Fabra, AURKA overexpression is driven by FOXM1 and MAPK/ERK activation in melanoma cells harboring BRAF or NRAS mutations: Impact on melanoma prognosis and therapy, J Invest Dermatol 137 (2017), 1297-1310.

[20] N. Yang, C. Wang, Z. Wang, S. Zona, S.X. Lin, X. Wang, M. Yan, F.M. Zheng, S.S. Li, B. Xu, L. Bella, J.S. Yong, E.W. Lam and Q. Liu, FOXM1 recruits nuclear Aurora kinase A to participate in a positive feedback loop essential for the self-renewal of breast cancer stem cells, Oncogene 36 (2017), 3428-3440.
[21] M. Yu, Z. Tang, F. Meng, M. Tai, J. Zhang, R. Wang, C. Liu and Q. Wu, Elevated expression of FoxM1 promotes the tumor cell proliferation in hepatocellular carcinoma, Tumour Biol $\mathbf{3 7}$ (2016), 1289-1297.

[22] G. Hu, Z. Yan, C. Zhang, M. Cheng, Y. Yan, Y. Wang, L. Deng, Q. Lu and S. Luo, FOXM1 promotes hepatocellular carcinoma progression by regulating KIF4A expression, J Exp Clin Cancer Res 38 (2019), 188.

[23] L. Zhou, Y. Du, L. Kong, X. Zhang and Q. Chen, Identification of molecular target genes and key pathways in hepatocellular carcinoma by bioinformatics analysis, Onco Targets Ther $\mathbf{1 1}$ (2018), 1861-1869.

[24] L. Zhang, Y. Huang, J. Ling, W. Zhuo, Z. Yu, M. Shao, Y. Luo and Y. Zhu, Screening and function analysis of hub genes and pathways in hepatocellular carcinoma via bioinformatics approaches, Cancer Biomark 22 (2018), 511-521.

[25] C. Liang, J. Zhao, H. Ge, G. Li and J. Wu, Clinicopathological and prognostic significance of FoxM1 in hepatocellular carcinoma patients: A meta-analysis, Onco Targets Ther 11 (2018), 3561-3571.

[26] L. Xia, W. Huang, D. Tian, H. Zhu, Y. Zhang, H. Hu, D. Fan Y. Nie and K. Wu, Upregulated FoxM1 expression induced by hepatitis $B$ virus $X$ protein promotes tumor metastasis and indicates poor prognosis in hepatitis B virus-related hepatocellular carcinoma, J Hepatol 57 (2012), 600-612.

[27] A. Alisi, M. Ghidinelli, A. Zerbini, G. Missale and C. Balsano, Hepatitis $\mathrm{C}$ virus and alcohol: same mitotic targets but differen signaling pathways, J Hepatol 54 (2011), 956-963.

[28] S.H. Giordano, S. Temin and N.E. Davidson, Systemic therapy for patients with advanced human epidermal growth factor receptor 2-positive breast cancer: ASCO clinical practice guideline update summary, J Oncol Pract 14 (2018), 501-504.

[29] G. Recondo, F. Facchinetti, K.A. Olaussen, B. Besse and L. Friboulet, Making the first move in EGFR-driven or ALKdriven NSCLC: First-generation or next-generation TKI? Nat Rev Clin Oncol 15 (2018), 694-708.

[30] J. Chen, H. Lu, W. Zhou, H. Yin, L. Zhu, C. Liu, P. Zhang, H. $\mathrm{Hu}$, Y. Yang and H. Han, AURKA upregulation plays a role in fibroblast-reduced gefitinib sensitivity in the NSCLC cell line HCC827, Oncol Rep 33 (2015), 1860-1866.

[31] D. Yan, X. Yan, X. Dai, L. Chen, L. Sun, T. Li, F. He, J. Lian and W. Cai, Activation of AKT/AP1/FoxM1 signaling confers sorafenib resistance to liver cancer cells, Oncol Rep $\mathbf{4 2}$ (2019), 785-796.

[32] Y. Zhi, M. Abudoureyimu, H. Zhou, T. Wang, B. Feng, R. Wang and X. Chu, FOXM1-mediated LINC-ROR regulates the proliferation and sensitivity to sorafenib in hepatocellular carcinoma, Mol Ther Nucleic Acids 16 (2019), 576-588.

[33] K. Zhang, T. Wang, H. Zhou, B. Feng, Y. Chen, Y. Zhi and R. Wang, A novel Aurora-A inhibitor (MLN8237) synergistically enhances the antitumor activity of sorafenib in hepatocellular carcinoma, Mol Ther Nucleic Acids 13 (2018), 176-188. 